\section{Patentiertes Molekül gegen Hautrötungen}

Der Wirkstoff Furfuryl Tetrahydropyranyladenine (Pyratine $\mathrm{XR}^{\circledast}$ ) aus der Familie der Cytokinine hat seine Wirksamkeit bei Rosazea-Symptomen in mehreren Studien unter Beweis gestellt. Auch langfristig sind Wirksamkeit und Sicherheit bestätigt (Tremaine AM et al., Journal of Drugs in Dermatology, June 2010, Vol 9, Issue 6:47-650): Nach 48 Behandlungswochen hat Pyratine $\mathrm{XR}^{\circledR}$ Erytheme um $44 \%$, Läsionen um $89 \%$ und Teleangiektasien um $27 \%$ reduziert.

Die Creme wird von der Haut rasch absorbiert, ist nicht reizend und für die langfristige Anwendung geeignet. Sie ist für normale, trockene und gemischte Haut bestimmt. Pyratine XR ${ }^{\oplus}$ Creme wird zwei Mal täglich aufgetragen und eignet sich auch als Make-up-Basis.

Nach Informationen von Nordesta

\section{Feuchtigkeitspflege für Lichtempfindliche}

Lichtempfindliche Patienten müssen sich jeden Tag vor UV-Strahlung schützen. Für diese Risikogruppe steht die täglich schützende Feuchtigkeitspflege Anthelios KA zur Verfügung. Die Kombination aus Pflege und UVSchutz eignet sich ideal für jeden Tag. Bei Einnahme von Medikamenten, die die Photosensibilität erhöhen, bestimmten Erkrankungen (z. B. aktinische Keratose, polymorphe Lichtdermatose) oder nach ästhetischen Behandlungen besitzt UV-Schutz eine besonders hohe Relevanz. Auch sehr helle Hauttypen sollten sich täglich und ganzjährig vor der Sonne schützen. Mit der Kombination von Feuchtigkeitspflege und UV-Schutz vereinfacht es La Roche-Posay diesen Personen nun, den Lichtschutz in ihren Tagesablauf zu integrieren.

Anthelios KA versorgt die Haut 24 Stunden mit Feuchtigkeit und schützt gleichzeitig vor UVA- und UVBStrahlung (LSF 100). Die pflegende Textur zieht schnell ein, fettet und klebt nicht.

Nach Informationen von La Roche-Posay

Phlebologenkonferenz in Asien

\title{
Krampfadertherapie überzeugt in Seoul
}

Die Elves Radial 2ring ${ }^{\mathrm{Tm}}$ - und Elves Radial slim $^{\mathrm{Tx}}$-Fasern waren Gegenstand klinischer Studien auf dem Chapter Meeting der UIP (Union Internationale de Phlébologie) in Seoul, das vom 27.-29.8.2015 stattfand. Zwei Studien hierzu wurden von Dr. Uldis Maurins, Riga, vorgestellt. Darin hat er dargelegt, dass Krampfaderbehandlungen mit Elves Radial ${ }^{\mathrm{TM}}$ schonend und effektiv sind: Bei der zweiphasigen und ringförmigen Abstrahlung der Fasern wird die Energie homogener an die Gefäßwand abgegeben. So kann die Vene noch gleichmäßiger verschlossen werden. Das Risiko für Perforationen der Venenwand ist dadurch minimiert.

Die Faser mit atraumatischer Faserspitze wird über eine kurze Einführschleuse direkt in die Vene geführt. Dank Ultraschall kann die Faserspitze überwacht und platziert werden.
In Kombination mit dem $1.470 \mathrm{~nm}$ Leonardo ${ }^{\circledast}$-Diodenlaser sind die Elves ${ }^{\circledR}$ Fasern die optimale Lösung für eine endovenöse thermische Ablation. Der Absorptionskoeffizient von Wasser in der Gefäßwand ist bei $1.470 \mathrm{~nm}$ größer als bei geringeren Wellenlängen von 810-1.064 nm. Deshalb kann die Laserenergie bei $1.470 \mathrm{~nm}$ besser kontrolliert werden, um thermische Reizungen der Gefäßwand zu vermeiden. Dazu Dr. Maurins: „Das Elves Radial $^{\mathrm{TM}}$-Laserverfahren minimiert im Vergleich zu dem auf Hämoglobin ausgerichteten 810-980-nm-Laser postoperative Schmerzen und Verletzungen aufgrund der 40-mal höheren Absorptionsrate in Wasser. Defekte Venen zeigten einen Tag nach der Therapie eine Reduktion des Venendurchmessers um 30-40\%.“

Nach Informationen von biolitec

\section{Umfassendes Medizinprodukt für den ästhetischen Anwendungsbereich}

Das Legend ${ }^{\circledast}$-System von Pollogen ist eine Methode, die es Fachkräften in der ästhetischen Dermatologie ermöglicht, alle Hautschichten zu behandeln und ein breites Spektrum kosmetischer Problemzonen abzudecken. Mit dem System können Verbesserungen der meisten Problemzonen im Gesichts- und Körperbereich erzielt werden. Gesichtsbehandlungen führen zu eindrucksvollen Resultaten, sowohl in Bezug auf Anti-Aging als auch hinsichtlich einer Verfeinerung des Hautbildes; auch zur Faltenminderung, Hautstraffung, Reduktion von Aknenarben und anderen Hautproblemen kann es eingesetzt werden.

Eine Körperkonturierung beinhaltet die Reduzierung lokaler Fettablagerungen, Cellulite-Behandlung, Verbesserung der Hautstruktur bei Dehnungsstreifen und Narben sowie eine Verringerung des Körperumfangs. Auch hier lassen sich gute Ergebnisse vorweisen. Die Behandlungen sind nahezu schmerzlos bei mini- maler Ausfallzeit. Patienten können bereits kurz nach der Behandlung zu ihrem Tagesgeschäft zurückkehren.

Für optimale Ergebnisse können drei ergänzende Technologien verwendet werden:

- Trilipo RF - zur Hautstraffung, sowie zur Cellulite- und Fettreduktion

- TriLipo DMA - um freigesetztes Körperfett zu beseitigen. Bei Gesichtsbehandlungen zum "Lifting“ des superfiziellens muskuloapneurotischen Systems

- VoluDerm HE - effizientes Verfahren zur Hautverjüngung

Nach Informationen von mi.to. pharm 